\title{
Rethought histologic classification of pleural mesothelioma to better treat: go forward from looking back
}

\author{
Philippe Astoul
}

Department of Thoracic Oncology, Pleural Diseases and Interventional Pulmonology - Aix-Marseille University, Marseille, France

Correspondence to: Philippe Astoul, MD, PhD. Department of Thoracic Oncology, Pleural Diseases and Interventional Pulmonology, Aix-Marseille University, Hôpital Nord, Chemin des Bourrely, 13015, Marseille Cedex 20, France. Email: pastoul@ap-hm.fr.

Provenance and Peer Review: This article was commissioned by the editorial office, Translational Lung Cancer Research. The article did not undergo external peer review.

Comment on: Nicholson AG, Sauter JL, Nowak AK, et al. EURACAN/IASLC Proposals for Updating the Histologic Classification of Pleural Mesothelioma: Towards a More Multidisciplinary Approach. J Thorac Oncol 2020;15:29-49.

Submitted Apr 23, 2020. Accepted for publication Jun 10, 2020.

doi: $10.21037 /$ tlcr-20-592

View this article at: http://dx.doi.org/10.21037/tlcr-20-592

Malignant pleural mesothelioma (MPM) is a rare, highly aggressive and deadly disease with a poor patient's lifeexpectancy. Most patients die within 2 years after the histologic diagnosis with a median survival of 7 to 10 months if untreated and a 5 -year survival rate of $5 \%$ (1). If few years ago, the main challenge has been the histologic diagnosis of this disease, at the present time the search of the best therapeutic strategy is in front line. However, an optimal management is not clearly defined yet despite growing efforts in the treatment armamentarium and research (2). These last years, initial management was roughly based on histological criteria represented by three major histologic subtypes of prognostic importance (epithelioid, biphasic, and sarcomatoid including desmoplastic features) usually clinically divided in two classes (epithelioid and non-epithelioid), surgery with curative intent, however only feasible in a minority of patients at the time of diagnosis, and systemic therapy, sometimes in a multidisciplinary setting. This therapeutic approach, mainly related to patient characteristics and clinical stage of the disease, failed. Therefore, mimicking the molecular and immunologic breakthroughs in the field of lung cancer, same advances are mandatory for the management of MPM. Mesothelioma WHO 2015 histologic classification, initial cornerstone for the management of the disease, is mainly based on resection specimens which is not the most frequent situation (3). Indeed if it is not recommended to make a diagnosis of mesothelioma based on cytology alone because of the high risk of diagnostic error, in case of suspicion, a confirmation is usually done by a tissue analysis ideally obtained by thoracoscopy route allowing representative material of the tumour (i.e., with sub-pleural fat and/or muscle to assess tumour invasion) in $90 \%$ of cases, and a complete visual examination of the pleura (4). In patients undergoing cytoreduction, including extended pleurectomy/ decortication (EPD) and extrapleural pneumonectomy (EPP), pleural specimens obtained by preoperative medical or surgical thoracoscopy have found a good histological correlation with the final post-operative diagnosis of mesothelioma subtype $(5,6)$. However, there is no standardization on the optimal number of biopsy specimens to characterize all the histological subtypes which is a major challenge due to the heterogeneity of this tumour. So far the recommendations for diagnosis still remain to take biopsies from separate areas of the pleural cavity usually guided by careful assessment during thoracoscopy after the realization of pre procedural imaging techniques. Among these late, if MRI and 18F-fluorodeoxyglucose (FDG)-PET provide information mainly for resectability, the detection of chest wall and diaphragmatic involvement and response to therapy, contrast-enhanced CT still remains the goldstandard for evaluation of MPM and initial diagnosis. However, the qualitative nature of this current approach is problematic in predicting the patient's outcome and the best therapeutic strategy. In an effort to skirt this drawback, 
quantitative image assessments are developing to better explore tumour volume, fissure invasion, and pleural thickness in general in order to improve the clinical staging and guide the treatment in particular the relevance of always controversial surgical approach in patients with localized or early-stage disease with a standardized system $(7,8)$. In case of diffuse MPM, patients with all histologic subtypes, if clinical eligibles, are considered for chemotherapy based on cisplatin and third-generation cytotoxic agent with or without bevacizumab (9) even other clinical trials using antiangiogenic agents have usually excluded sarcomatous sub-types $(10,11)$. Recently, in mirror on the breakthroughs for lung cancer treatment, clinical phase I/II trials using immune checkpoint inhibitors have shown interesting results in the treatment of MPM and drugs targeting programmed death 1 (PD-1)/PD-L1 alone or combined with cytotoxic T-lymphocyte-associated protein 4 (CTLA-4) showed efficacy in all histologic subtypes (12). However, few countries have given regulatory approval and immunotherapy for the treatment of MPM is usually offlabel use as salvage therapy. From these simple comments concerning the management of mesothelioma in recent years, the need to revisit the histologic classification emerges based on validated clinical and translational data useful for therapeutic management, clinical trials, and research investigation. Recently, a multidisciplinary group including pathologists, molecular biologists, radiologists, surgeons, and oncologists sponsored by European Network for Rare Adult Solid Cancers (EURACAN)/International Association of the Study of Lung Cancer (IASLC), met to confront their clinical experiences according to the current histologic classification in an effort to optimize a future clinical management of MPM and research in this field (13). Regarding the pathology aspect of the disease, a focus has been done on: (I) the diagnosis of localized MPM with a potential radical surgical treatment with complete resection requiring careful preoperative imaging assessment and surgical findings. The differential diagnosis with a welldifferentiated papillary mesotheliomas (WDPMs), which do not harbor alterations of BRCA1 associated protein 1 (BAP1), cyclin-dependent kinase inhibitor 2A (CDKN2A), $\mathrm{NF} 2$ and SETD2 genes, is of importance to rule out invasive diffuse mesothelioma (14,15); (II) among the three well-known mesothelioma subtypes, rare features seen in epithelioid or sarcomatoid mesothelioma concerning different architectural patterns, cytologic features and stromal characteristics which have an impact on patient's prognosis; (III) MPM in situ (MMIS), may be a starting point for diffuse MPM, which is difficult to distinguish from reactive/atypical mesothelial proliferation. In absence of invasion, loss of BAP1 expression by IHC and/or the presence of a homozygous deletion of CDKN2A (p16) by FISH or by methylthioadenosine phosphorylase (MTAP) IHC allow a differential diagnosis between neoplastic and non-neoplastic cells (16-18). Therefore the diagnosis of MMIS should be made in patient with pleural effusion but without clinical and radiologic evidence of pleural tumour and based on multiple pleural biopsies with a mesothelial proliferation limited to the serosal surface with BAP1 loss and/or CDKN2A/p16 homozygous deletion; (IV) the routinely application of a pathologic grading system (PGS) to pleural biopsies and resection specimens in case of epithelial mesothelioma in order to determine risk categories of patients, therapeutic options, and potential clinical trials (19). The risk stratification according to the literature seems to be better approached with a two-tier system of low and high grade based on nuclear atypia (1 for mild, 2 for moderate, 3 for severe), mitotic activity [ 1 for low, 2 for intermediate (2-4 per $\left.2 \mathrm{~mm}^{2}\right), 3$ for high], and the presence or absence of necrosis (20). As time goes by, from the lung cancer experience, we are getting more and more familiar with biotherapy and it potential benefit in cancer patients based on the presence of biomarkers. Nevertheless, despite recent advances, the molecular characteristics of MPM is poorly understood. If the PD-L1 status, loss of BAP1, and CDKN2A deletion, which may orientate the decision-making therapeutic strategy, should be part of histologic reports, the others molecular analyses have to be incorporated to future trials as prognostic parameters. A continuous model of molecular characteristics in mesothelioma patients (different molecular cluster groups), recapitulating the epithelial-to-mesenchymal transition, recently showed a good correlation with the prognosis with variations depending on immune and vascular pathways $(20,21)$. In these molecular cluster groups, claudin 15 (CLDN15) and VIM were the most significantly upregulated genes in the epithelioid and sarcomatoid sub-types allowing to use the $\log 2$ CLDN15/ VIM ratio to distinct different subgroups and their specific prognosis (22). Regarding blood-based biomarkers in the field of MPM, they remain useless for diagnosis and prognosis even several teams use soluble mesothelin-related peptides (SMRP) to monitor the disease after or during specific treatment (23). In summary, despite considerable efforts devoted to understanding and better treating MPM, clinical practice has not changed and chemotherapy, based 
on cisplatin and third-generation cytotoxic agent with or without bevacizumab, still remains the standard option. Hopefully after significant success with personalized therapy in other solid tumor types, in particular lung cancer, a paradigm shift to personalized therapy and precision medicine is becoming of interest for the management of patients with MPM. We are in the midst of an exciting time and, although MPM treatment has lagged behind that of other cancer types, biotherapy have recently shown preliminary efficacy, and even these results need to be confirmed in larger clinical trials, there is a trend to move toward the application of molecular tests in biopsy specimens not only for therapeutic strategy but also for prognosis purpose. Consequently, as mentioned by Nicholson and co-workers (13), standardization of adequate tissue sampling to diagnose and molecularly characterize MPM is paramount because more targeted treatment options will become available in a near future, testing for multiple markers will be required. Abundant and good quality pleural samples will need to be acquired. At the era of liquid biopsy, a revolutionary technique that is opening previously unexpected perspectives, biopsy samples, ideally done by thoracoscopy route (24), are necessary. It still remains clear that for the management of MPM 'the tissue is the issue'.

\section{Acknowledgments}

The author is indebted to Coline Astoul for her careful review of the manuscript.

Funding: None.

\section{Footnote}

Conflicts of Interest: The author has completed the ICMJE uniform disclosure form (available at http://dx.doi. org/10.21037/tlcr-20-592). The author has no conflicts of interest to declare.

Ethical Statement: The author is accountable for all aspects of the work in ensuring that questions related to the accuracy or integrity of any part of the work are appropriately investigated and resolved.

Open Access Statement: This is an Open Access article distributed in accordance with the Creative Commons Attribution-NonCommercial-NoDerivs 4.0 International License (CC BY-NC-ND 4.0), which permits the non- commercial replication and distribution of the article with the strict proviso that no changes or edits are made and the original work is properly cited (including links to both the formal publication through the relevant DOI and the license). See: https://creativecommons.org/licenses/by-nc-nd/4.0/.

\section{References}

1. Tsao AS, Lindwasser OW, Adjei AA, et al. Current and Future Management of Malignant Mesothelioma: A Consensus Report from the National Cancer Institute Thoracic Malignancy Steering Committee, International Association for the Study of Lung Cancer, and Mesothelioma Applied Research Foundation. J Thorac Oncol 2018;13:1655-67.

2. Astoul P, Roca E, Galateau-Salle F, et al. Malignant pleural mesothelioma: from the bench to the bedside. Respiration. 2012;83:481-93.

3. WHO Classification of Tumours of the Lung, Pleura, Thymus and Heart. Lyons, France: International Agency for Research on Cancer (IARC), 2015.

4. Scherpereel A, Astoul P, Baas P, et al. Guidelines of the European Respiratory Society and the European Society of Thoracic Surgeons for the management of malignant pleural mesothelioma. Eur Respir J 2010;35:479-95.

5. Greillier L, Cavailles A, Fraticelli A, et al. Accuracy of pleural biopsy using thoracoscopy for the diagnosis of histologic subtype in patients with malignant pleural mesothelioma. Cancer 2007;110:2248-52.

6. Chirieac LR, Hung YP, Foo WC, et al. Diagnostic value of biopsy sampling in predicting histology in patients with diffuse malignant pleural mesothelioma. Cancer 2019;125:4164-71.

7. Gill RR, Yeap BY, Bueno R, et al. Quantitative Clinical Staging for Patients With Malignant Pleural Mesothelioma. J Natl Cancer Inst 2018;110:258-64.

8. Friedberg JS, Culligan MJ, Tsao AS, et al. A Proposed System Toward Standardizing Surgical-Based Treatments for Malignant Pleural Mesothelioma, From the Joint National Cancer Institute-International Association for the Study of Lung Cancer-Mesothelioma Applied Research Foundation Taskforce. J Thorac Oncol 2019;14:1343-53.

9. Zalcman G, Mazieres J, Margery J, et al. Bevacizumab for newly diagnosed pleural mesothelioma in the Mesothelioma Avastin Cisplatin Pemetrexed Study (MAPS): a randomised, controlled, open-label, phase 3 trial. Lancet 2016;387:1405-14.

10. Grosso F, Steele N, Novello S, et al. Nintedanib plus 
pemetrexed/cisplatin in patients with malignant pleural mesothelioma: phase II results from the randomized, placebo-controlled LUME-Meso trial. J Clin Oncol 2017;35:3591-600.

11. Scagliotti GV, Gaafar R, Nowak AK, et al. Nintedanib in combination with pemetrexed and cisplatin for chemotherapy-naive patients with advanced malignant pleural mesothelioma (LUME-Meso): a double-blind, randomised, placebo-controlled phase 3 trial. Lancet Respir Med 2019;7:569-80.

12. Forde PM, Scherpereel A, Tsao AS. Use of immune checkpoint inhibitors in mesothelioma. Curr Treat Options Oncol 2019;20:18.

13. Nicholson AG, Sauter JL, Nowak AK, et al. EURACAN/IASLC Proposals for Updating the Histologic Classification of Pleural Mesothelioma: Towards a More Multidisciplinary Approach. J Thorac Oncol 2020;15:29-49.

14. Sun M, Zhao L, Weng Lao I, et al. Well-differentiated papillary mesothelioma: a 17 -year single institution experience with a series of 75 cases. Ann Diagn Pathol 2019;38:43-50.

15. Stevers M, Rabban JT, Garg K, et al. Well-differentiated papillary mesothelioma of the peritoneum is genetically defined by mutually exclusive mutations in TRAF7 and CDC42. Mod Pathol 2019;32:88-99.

16. Bruno R, Ali G, Fontanini G. Molecular markers and new diagnostic methods to differentiate malignant from benign mesothelial pleural proliferations: a literature review. J Thorac Dis 2018;10 S342-52.

17. Churg A, Galateau-Salle F, Roden AC, et al. Malignant mesothelioma in situ: morphologic features and clinical outcome. Mod Pathol 2020;33:297-302.

18. Quetel L, Meiller C, Assié JB, et al. Genetic alterations of malignant pleural mesothelioma: association with tumor heterogeneity and overall survival. Mol Oncol 2020;14:1207-23.

19. Pelosi G, Papotti M, Righi L, et al. Pathologic grading of malignant pleural mesothelioma: an evidence-based proposal. J Thorac Oncol 2018;13:1750-61.

20. Rosen LE, Karrison T, Ananthanarayanan V, et al. Nuclear grade and necrosis predict prognosis in malignant epithelioid pleural mesothelioma: a multi-institutional study. Mod Pathol 2018;31:598-606.

21. Hmeljak J, Sanchez-Vega F, Hoadley KA, et al. Integrative molecular characterization of malignant pleural mesothelioma. Cancer Discov 2018;8:1548-65.

22. Alcala N, Mangiante L, Le-Stang N, et al. Redefining malignant pleural mesothelioma types as a continuum uncovers immune-vascular interactions. EBioMedicine 2019;48:191-202.

23. Nicolini F, Bocchini M, Bronte G, et al. Malignant Pleural Mesothelioma: State-of-the-Art on Current Therapies and Promises for the Future. Front Oncol 2020;9:1519.

24. Skalski JH, Astoul PJ, Maldonado F. Medical thoracoscopy. Semin Respir Crit Care Med 2014;35:732-43.
Cite this article as: Astoul P. Rethought histologic classification of pleural mesothelioma to better treat: go forward from looking back. Transl Lung Cancer Res 2020;9(4):1613-1616. doi: $10.21037 /$ tlcr-20-592 